\title{
An Explainable approach to Deep Learning from CT-scans for Covid Identification
}

\author{
Eduardo Soares, Plamen Angelov, Fellow, IEEE, Ziyang Zhang
}

\begin{abstract}
The Covid-19 disease has spread widely over the whole world since the beginning of 2020 . Following the epidemic which started in Wuhan, China on January 30, 2020 the World Health Organization (WHO) declared a global health emergency and a pandemic. Researchers of different disciplines work along with public health officials to understand the SARSCoV-2 pathogenesis and jointly with the policymakers urgently develop strategies to control the spread of this new disease. Recent findings have observed specific image patterns from computed tomography (CT) for patients infected by SARSCoV-2 which are distinct from the other pulmonary diseases. In this paper, we propose an explainable-by-design that has an integrated image segmentation mechanism based on SLIC that improves the algorithm performance and the interpretability of the resulting model. In order to evaluate the proposed approach, we used the SARS-CoV-2 CT scan dataset that we published recently and has been widely used in the literature. The proposed Super-xDNN could obtain statistically better results than traditional deep learning approaches as DenseNet-201 and Resnet-152. Furthermore, it also improved the explainability and interpretability of its decision mechanism when compared with the $x D N N$ basis approach that uses the whole image as prototype. The segmentation mechanism of Super-xDNN favored a decision structure that is more close to the human logic. Moreover, it also allowed the provision of new insights as a heat-map which highlights the areas with highest similarities with Covid-19 prototypes, and an estimation of the area affected by the disease.
\end{abstract}

Index Terms-Explainable AI, prototype-based models, image segmentation, superpixels, Covid-19 identification.

\section{INTRODUCTION}

$\mathbf{I}_{3}$ N December 2019, an outbreak coronavirus (SARS-CoV2) infection began in Wuhan, the capital of central China's Hubei province [1]-[3] causing a new coronavirus disease known as Covid-19. Since then, this disease became one of the most rapidly spreading infectious diseases, affecting a large number of the population globally. On January 30, 2020 the World Health Organization (WHO) declared a global health emergency [4]. The Covid-19 infection critically affects the respiratory systems creating lesions that affects the normal functioning of the lungs.

The most common symptoms of Covid-19 are dry cough, tiredness, mild to moderate respiratory illness, loss of taste sensation, and fever [5]. However, serious symptoms as difficulty breathing or shortness of breath, chest pain, and loss of

\footnotetext{
* Corresponding author
}

E. Soares*, P. Angelov an Z. Zhang are with LIRA, School of Computing and Communications, Lancaster University, Lancaster, LA1 4WA, UK, p.angelov@lancaster.ac.uk, z.zhang51@lancaster.ac.uk,e.almeidasoares@lancaster.ac.uk. speech or movement are also identified [6]. Patients that have previous medical complications as obesity, diabetes, chronic respiratory disease, cardiovascular diseases, and cancer are more likely to develop the serious form of the disease. Another factor that may be negatively considered to disease is the age. Elderly patients are more sensitive to the serious form of the Covid-19 disease than young age groups [7].

The Covid-19 infection may cause severe impacts on the respiratory systems. Therefore, medical imaging as computed tomography (CT) can reveal specific image patterns features that differs Covid-19 from other pulmonary diseases. For example, patients diagnosed with Covid-19 revealed bilateral lung opacities $98 \%$ of chest CTs in infected patients in Wuhan [8]. Findings described lobular and sub-segmental areas of consolidation as the most typical patterns [8]. High rates of ground-glass opacities and consolidation, sometimes with a rounded morphology and peripheral lung distribution are also reported [9], [10]. Therefore, thoracic radiology evaluation is often key to the evaluation of patients suspected of COVID-19 infection [11].

Moreover, CT-scans also presents advantages over X-ray in terms of Covid-19 identification and analysis as they present convenient examination of disease, and its location [12] whereas X-ray allows the examination of dense tissues only [13]. Therefore,for Covid-19 detection, a CT-scan of the chest is attracting the researchers' attention [14]. In this sense, different approaches using artificial intelligence, more specifically, deep learnings have been proposed for Covid19 identification via medical imaging [15]. However, despite of the great performance achieved by theses approaches on the Covid-19 identification task, they suffer of lack of explainability as they are "black-box", and as a consequence their results are not reliable [16]. Medical decisions are very critical and needs to rely on auditable and transparent systems [17].

Therefore, in this paper we propose a new explainable-bydesign approach that uses patches (super-pixels) of CT-scans in order to make its decision. The proposed method provides a transparent structure based on prototypes that allows users to investigate and audit its decisions. To evaluate the proposed approach we use the dataset of CT-scans for SARS-CoV2 identification that we published earlier [18]. The dataset contains CT-scans of 120 patients divided between 60 patients diagnosed with Covid-19 and 60 patients non-infected by Covid-19. The proposed method offers clear explainability by its design. 


\section{BACKGROUND REVIEW}

Recent advances in medical science have significantly improved the health care system over the last decades [19]. A powerful stream of such advances is based on the artificial intelligence and, more specifically, on the deep learnings [20].

Such technologies assist medical doctors in diagnosing and treating patients. However, the effectiveness of such algorithms are dependent on the availability of data and their quality [21]. Deep leanings achieved highly accurate results in terms of disease prediction and detection. Applications of deep leanings have introduced new breakthroughs in the field of healthcare making use of the various sources of medical data, such as Magnetic Resonance Imaging (MRI), X-rays, Positron Emission Tomography (PET), Computerized Tomography (CT) scans [22]. In the medical domain, deep learnings has been applied to a wide range of sub-disciplines such as ophthalmology [23], neurology [24], cancer detection [25], and cardiology [26]. However, one of the unresolved research challenges in deep learning which is critically important for medical image analysis is the ability to clearly explain its decision process in a human-understandable manner [19]. The main challenge for the wider acceptance of deep learning based models is the lack of transparency of their decisions especially for high stake applications such as the medical ones [19], [27], [28]. In this paper, we offer a new explainable-by-design approach as a step to address this unsolved challenge.

\section{Proposed Approach}

Here, we introduce the proposed Super-xDNN. Differently from the recently introduced xDNN approach [29] that uses the whole image as prototype, the proposed Super-xDNN is equipped with a mechanism that allows segmentation of a given image that possibilities to have specific patches of the images as prototypes. In this sense, it improves the explainability of the generated model as specialists can check specific regions the images that influenced the algorithm's decision. Moreover, it also possibilities to estimate the size of the area used for the decision-making. The Super-xDNN approach is described as a feedforward neural network. The training architecture that is composed by the following layers:

1) SLIC Superpixel segmentation;

2) Features layer;

3) Density layer;

4) Conditional probability layer;

5) Prototype identification layer;

It is important to highlight that the Super-xDNN is done per class. Thus, Super-xDNN is constituted of multiples structures for each class as illustrated by Fig. (positive Covid19 class).

1) SLIC Superpixel segmentation: The first step of the Super-xDNN algorithm is composed by an image segmentation layer which uses the SLIC Superpixel [30] algorithm to accomplish this task. The SLIC algorithm uses an adapted version of the $k$-means clustering approach to segment images and generate superpixels [31]. In this sense, it does not require much computational power. Despite its simplicity, SLIC has proven to be faster and improve segmentation performance compared to other graph-based and Gradientascent-based algorithms [31]. Although we use the SLIC superpixel algorithm in this paper, the Super-xDNN structure is flexible, and the segmentation layer can be adapted to other algorithms as Mask R-CNN [32] and YoLo [33].

Superpixels for positive classes (classes that we want to identify) may require labeling (see Fig. 1).

2) Features layer: The feature Layer is responsible for the global features vector extraction from the superpixels. It can be formed by a fully connected layer of a convolutional neural network approach such as AlexNet [34], VGG-16 [35], and Inception [36], residual neural networks as Resnet [37] and, Inception-Resnet [38], and Transformers-based approaches [39]. The aforementioned approaches allows the extraction of more abstract and discriminative high-level features. The extracted features have direct impact on the performance of the algorithm. Therefore, an optimized set of features may be required for specific problems. Super-xDNN also allows the inclusion of features generated by traditional as GIST [40] or HoG [41] can also form this feature layer.

In this paper, we consider the training dataset as $x=$ $\left\{x_{1}, \ldots, x_{N}\right\} \in \mathbb{R}^{n}$ with corresponding class labels $y_{1}, \ldots, y_{C}$ $\in\{1, \ldots, C\}$. Where, $N$ is the number of training data samples and $n$ is the dimensionality (number of features); $m$ is the number of superpixels per images; $C$ is the number of classes. The most descriptive data samples are selected as prototypes $\pi \in P \subset X$ for each class. Where, $M_{j}$ denotes the total number of prototypes of class $j ; M_{j}=\left|P_{j}\right|$; $M=\sum_{j=1}^{C} M_{j}$. We consider more than a single prototype per class, so $M_{j}>1$ for $\forall j$.

Prototypes are the most representative data samples of the training dataset. Then, any new data sample, $x \in \mathbb{R}^{n}$ can be associated with the nearest prototype from the sets $P_{1}, P_{2}, \ldots, P_{C} ; P=P_{1} \cup P_{2} \cup \ldots \cup P_{C}$.

$$
L(x)=\underset{x \in X}{\operatorname{argmin}} \min _{\pi \in P} d(x, \pi) .
$$

3) Density layer: The density layer is composed of neurons which have as activation function the data density $D$. This layer defines the mutual proximity of the data samples in the data space. The data density is represented by the following Cauchy function [42]:

$$
D(x)=\frac{1}{1+\frac{\|x-\mu\|^{2}}{\|\sigma\|^{2}}},
$$

where $D$ is the density, $\mu$ is the global mean, and $\sigma$ is the variance. [42] demonstrated theoretically that mutual proximity of the data samples in the data space and using Euclidean (or Mahalanobis) type distance $D$ takes the form of a Cauchy function.

Density can also be updated recursively [43]:

$$
D\left(x_{i}\right)=\frac{1}{1+\left\|x_{i}-\mu_{i}\right\|^{2}+\sum_{i}-\left\|\mu_{i}\right\|^{2}} .
$$




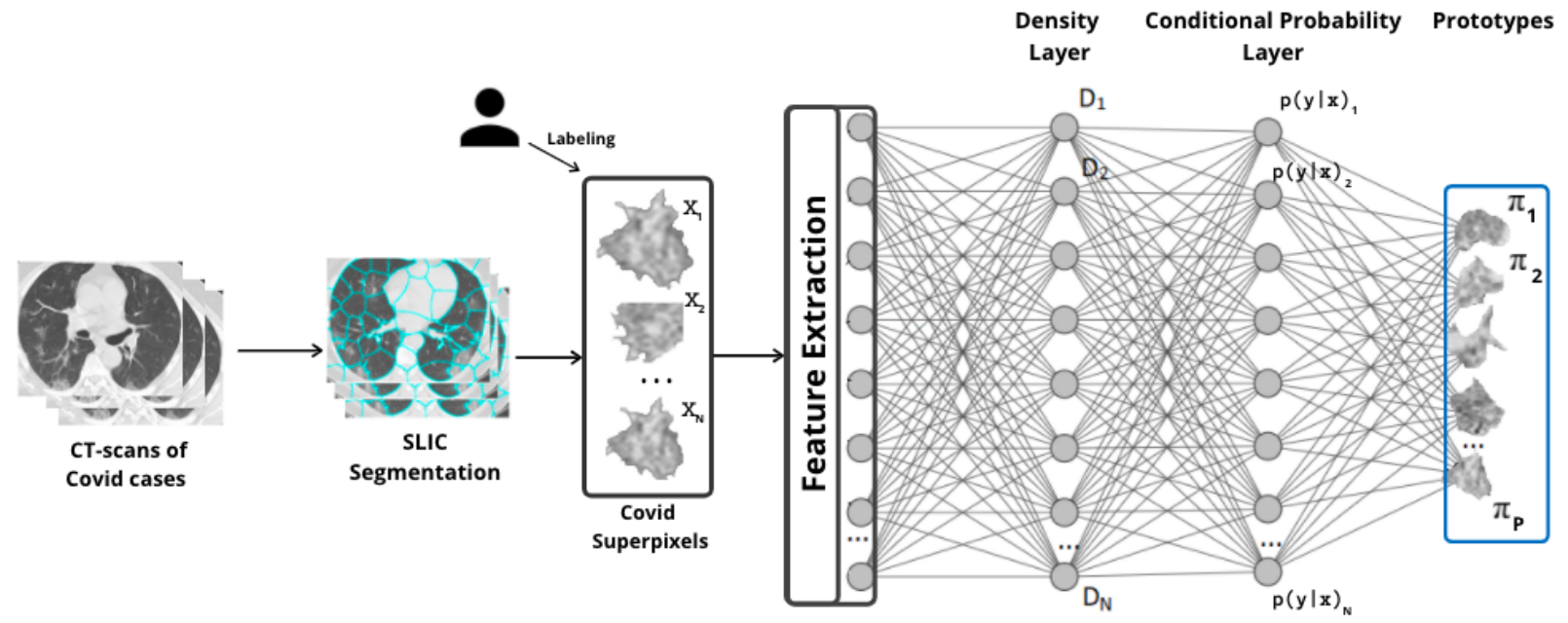

Fig. 1. Training architecture for the positive Covid-19 class. Labeling of the positive classes may be necessary.

where $i=1, \ldots, N, \mu$ and the scalar product, $\sum$ can be updated recursively as follows:

$$
\begin{gathered}
\mu_{i}=\frac{i-1}{i} \mu_{i-1}+\frac{1}{i} x_{i}, \\
\sum_{i}=\frac{i-1}{i} \sum_{i-1}+\frac{1}{i}\left\|x_{i}\right\|^{2} \quad \sum_{1}=\left\|x_{1}\right\|^{2} .
\end{gathered}
$$

The data density, $D$ denotes the closeness of a data sample to the mean, $\mu$. If the values are normalized between 0 and 1 the density has the range $0<D \leq 1$. Where $D=1$ when $x=\mu$. Data samples that are closer to the global mean have higher density values. The data density value indicates how strongly a particular data sample is influenced by other data samples in the data space due to their mutual proximity. In this sense, data density $D$ indicates the centrality of a data sample in the data space and its eligibility to become a prototype.

4) Conditional probability layer: The conditional probability layer, also known as typicality, $\tau$, is estimated from the empirically observed data as described in [42]. The typicality is given by eq. (6), where integral of $\int_{-\infty}^{\infty} p(C \mid x) d x=1$ is multi-modal version of pdf [42]:

$$
p(y \mid x)=\frac{\sum_{i=1}^{M} N_{i} D(x)}{\sum_{i=1}^{M} N_{i} \int_{-\infty}^{\infty} D(x) d x}
$$

where $N_{i}$ denotes the number of data samples associated with the $i-$ th data cloud, $\sum_{i=1}^{C} ; N_{i}=N$.

As $p(C \mid x)$ is empirically derived, it does not rely on any prior assumption about the data distribution type or even about the random or deterministic nature of the data [42].

5) Prototypes layer: The Super-xDNN method is trained per class as illustrated by Fig. 1. Therefore, all the calculations are done for each class separately. Prototypes are defined as the local peaks of the data density and are independent from each other. Thus, the Super-xDNN allows the prototypes set to be modified (prototypes can be added or removed) without influencing the other existing ones. As the method is trained per class, it can be parallelizable and suitable for applications as federated learning.

Super-xDNN assignes data samples to the nearest identified prototype as follows:

$$
j^{*}=\underset{i=1, . ., N ; j=1, . ., M}{\operatorname{argmin}}\left\|x_{i}-\pi_{j}\right\|^{2}
$$

The area of influence of the identified prototypes are known as data clouds [42]. Because their shape is not regular (e.g., hyper-spherical, hyper-ellipsoidal, etc.) and the prototype is not necessarily the statistical and geometric mean, but actual data sample [42].

If the following condition is met, then new prototypes are added to the set of prototypes [42]:

$$
\begin{gathered}
\text { IF }\left(D(x) \geq \max _{j=1, . ., M} D\left(\pi_{j}\right)\right) \\
\text { OR } \quad\left(D(x) \leq \min _{j=1, . ., M} D\left(\pi_{j}\right)\right)
\end{gathered}
$$

THEN (add a new data cloud $(j \leftarrow j+1)$ )

6) Learning Procedure: The learning mechanism for the proposed approach is summarised by the following pseudocode.

\section{Super-xDNN: Learning Procedure}

1: Segment and extract superpixels using the SLIC algorithm;

2: Extract the feature vectors from the superpixels using a fully connected layer;

3: Read the first feature vector sample $x_{i}$ of class $c$;

4: Normalise the data as detailed in [29]

5: Set $i \leftarrow 1 ; j \leftarrow 1 ; \pi_{1} \leftarrow x_{i} ; \mu \leftarrow x_{1} ; N \leftarrow 1$

6: FOR $i=2, \ldots$

7: $\quad$ Read $x_{i}$; 
8: Calculate $D\left(x_{i}\right)$ and $D\left(\pi_{j}\right)(j=1,2, \ldots, M)$ according to eq. (2);

9: $\quad$ IF eq. (8) holds

10: $\quad$ Create new prototype: $j \leftarrow j+1 ; \pi_{j} \leftarrow x_{i} ; N \leftarrow N+1$

11: ELSE

12: $\quad$ Search for the nearest prototype according to eq. (7);

13: Update the nearest prototype as:

$$
\begin{array}{ll} 
& N \leftarrow N+1 ; \\
& \leftarrow \leftarrow \frac{N_{j}}{N_{j}+1} \pi_{j}+\frac{N_{j}}{N_{j}+1} x_{i} ; \\
\text { 14: } \quad \text { END } & \\
\text { 15: } & \text { END }
\end{array}
$$

\section{A. Architecture and Validation of $x D N N$}

The architecture for the validation phase of the $\mathrm{xDNN}$ method is illustrated by Fig. 2 .

The validation process of $\mathrm{xDNN}$ is composed of the following layers:

1) Segmentation layer;

2) Features layer;

3) Local decision-making;

4) Global decision-making;

5) Estimated affected area.

1) Segmentation layer: This layer is responsible to extract superpixels from the new arrival samples. In this case, we also use the SLIC algorithm for this task.

2) Features layer: This layer is similar to the features extraction layer described and used in the training process.

3) Local decision-making (decision per superpixel): :

This layer provides the degree of similarity, $S$, between an unlabeled superpixel and the respective nearest prototype. The similarity between any new superpixel and a prototype can be determined by a SoftMax-like equation (9).

$$
\lambda\left(Y=x_{i} \mid \pi_{j}\right)=\frac{S_{j}}{\sum_{j=1}^{M} S_{j}},
$$

where,

$$
\begin{gathered}
S_{j}=S\left(x_{i}, \pi_{j}\right)=\frac{1}{1+\frac{\left\|x_{i}-\pi_{j}\right\|^{2}}{\left\|\sigma_{j}\right\|^{2}}}, \\
J=-\log \lambda\left(Y=x_{i} \mid \pi_{j}\right) .
\end{gathered}
$$

where $Y$ is the $j-t h$ validation superpixel sample. $S$ is the degree of similarity between the unlabeled data sample and the respective prototype. The label for each superpixel is obtained by the following equation (12):

$$
\text { label }=\underset{c=1,2, \ldots, C}{\operatorname{argmax}}\left(\lambda_{c}^{*}\right)
$$

4) Global decision-making (final decision): The global decision-making is responsible to provide the final label to the image. In this case, the final label is considered based on the positive classes identified during the local decisionmaking. If the identified positive classes have neighbours the "Covid-19" label is attached. On the other hand, if just a single superpixel is positively identified, this is considered as an outlier and ignored.

5) Estimated affected area: The estimation of the affected can be inferred by:

$$
\text { Affected Area }(\%)=\frac{\text { Infected superpixels }}{\text { Total superpixels }} \times 100
$$

\section{Covid DatA}

To evaluate our proposed Super-xDNN approach, we consider the Sars-CoV-2 CT-scan dataset [18] that we recently introduced and has widely used since then. The dataset is composed of 2482 CT-scans slices, which is divided between 1252 for patients diagnosed with Covid-19, and $1230 \mathrm{CT}$ scans slices for patients not diagnosed with the disease, but presented other pulmonary diseases. These data has collected

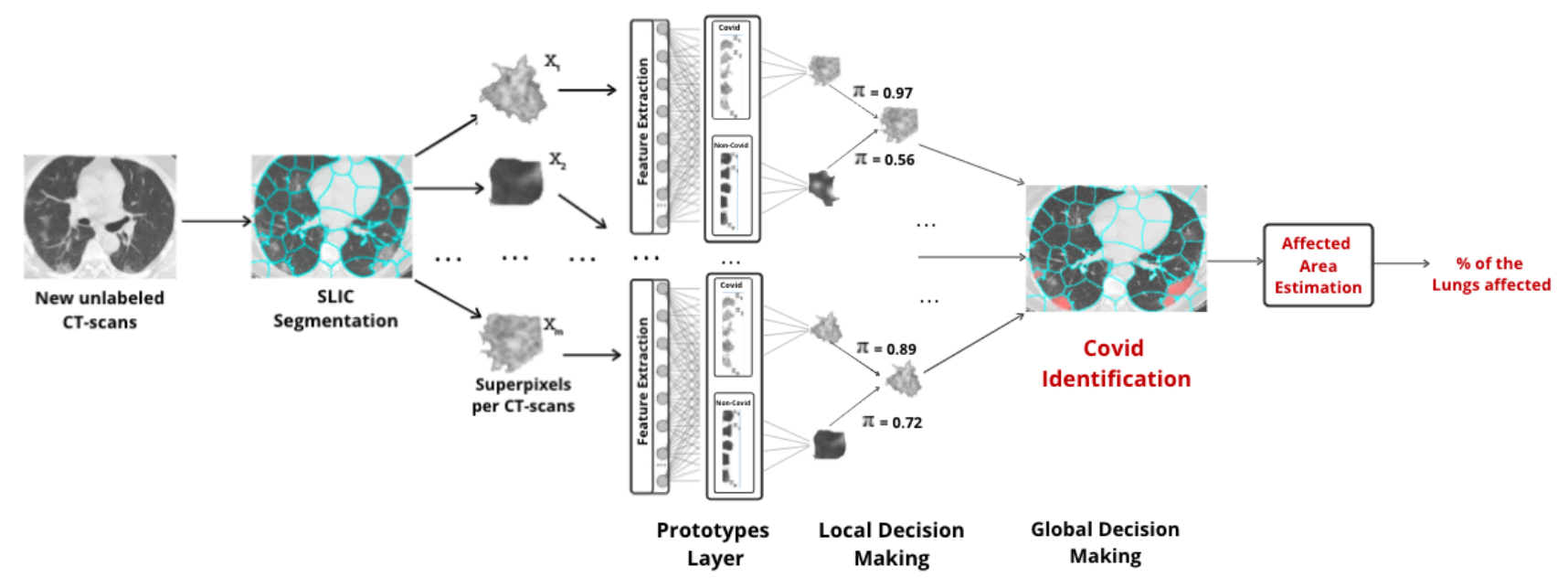

Fig. 2. Architecture for the validation process of the proposed xDNN. 
from March 15 to April 152020 in the Public Hospital of the Government Employees of Sao Paulo - Brazil. The detailed number of patients is illustrated by Table I.

TABLE I

COVID-19 DATASET DISTRIBUTION

\begin{tabular}{c|cc}
\hline Patients & Infected & non-Infected \\
& & \\
\hline Male & 32 & 30 \\
Female & 28 & 30 \\
Total & 60 & 60 \\
\hline
\end{tabular}

The patients inclusion criteria for this dataset includes the following requisites:

- Patients with a positive new coronavirus nucleic acid antibody and confirmed by the CDC;

- Patients who underwent thin-section CT;

- $\operatorname{Age} \geq 18$

- Presence of lung infection in CT images.

The SLIC Superpixels method has been employed to segment the lungs regions. The SLIC method applies the $k$-means algorithm to generate patches of images called superpixels [31]. The parameter $k$ defines the number of superpixels identified on a image [31], and the parameter $m$ denotes to the compression control the regions [31]. As in the parameters have been defined as $k=200$ and $m=30$ [44]. A CT-scan segmented by the superpixel technique is illustrated by Fig. 3 .

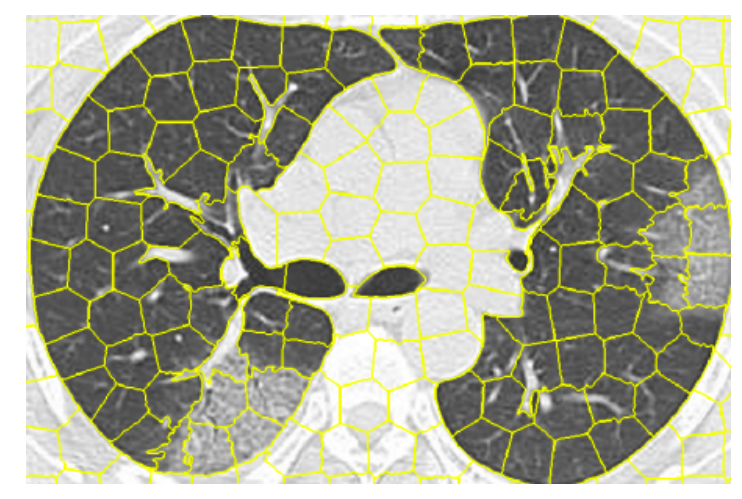

Fig. 3. CT-scan slice segmented into superpixels.

10000 superpixels have been randomly selected for Covid patients and 10000 superpixels have been selected for nonCOVID patients [44]. Fig. 4 illustrates superpixels extracted trough the SLIC method.

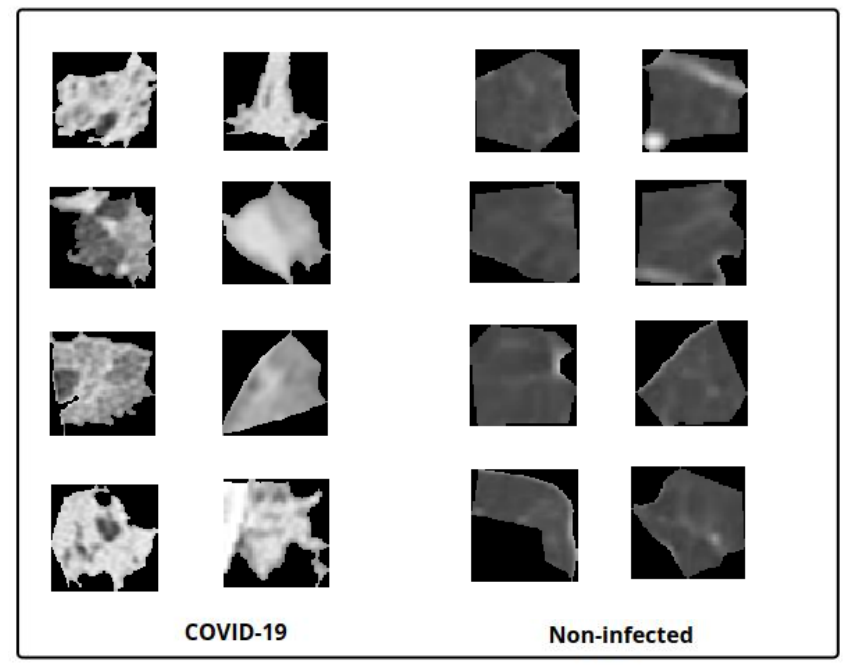

Fig. 4. Extracted superpixels.

\section{A. Performance Evaluation}

In order to evaluate the proposed approach the following metrics have been considered:

Accuracy:

$$
\operatorname{Accuracy}(\%)=\frac{T P+T N}{T P+F P+T N+F N} \times 100,
$$

Precision:

$$
\operatorname{Precision}(\%)=\frac{T P}{T P+F P} \times 100,
$$

Recall:

$$
\operatorname{Recall}(\%)=\frac{T P}{T P+F N} \times 100,
$$

F1 Score:

$$
\text { F1 Score }(\%)=2 \times \frac{\text { Precision } \times \text { Recall }}{\text { Precision }+ \text { Recall }} \times 100,
$$

where $T P, F P, T N, F N$ denote true and false, negative and positive respectively.

All the experiments were conducted with the Google Colaboratory platform. We used Tesla V100-SXM2-16GB GPU for feature extraction and Intel(R) Xeon(R) CPU $2.30 \mathrm{GHz}$ $\mathrm{CPU}$ to train the Super-xDNN model and evaluate it.

\section{B. Results}

In this section we report the results obtained by the proposed Super-xDNN approach. The dataset has been divided into $80 \%$ for training purposes and $20 \%$ for validation purposes. Results presented in Table II compare the performance of the Super-xDNN algorithm with other state-of-the-art approaches, including the xDNN approach and mainstream (black-box) deep neural networks.

The Super-xDNN approach demonstrated higher results in terms of accuracy, precision, and F1-score than the other 
TABLE II

RESULTS CONSIDERING DIFFERENT METHODS FOR THE COVID-19 IDENTIFICATION

\begin{tabular}{c|ccccc}
\hline Metric & Accuracy & Precision & Recall & F1 Score & Time (s) \\
\hline Method & $\underline{\mathbf{9 8 . 9 \%}}$ & $\underline{\mathbf{9 9 . 5 \%}}$ & $\underline{\mathbf{9 8 . 3 \%}}$ & $\mathbf{9 8 . 9 \%}$ & 210 \\
Super-xDNN & $97.38 \%$ & $99.16 \%$ & $\mathbf{9 5 . 5 3 \%}$ & $\underline{96.42 \%}$ & $\mathbf{1 1 . 8 2}$ \\
xDNN [18] & $96.5 \%$ & $96.5 \%$ & $96.5 \%$ & $96.5 \%$ & 13731 \\
DenseNet-201 [44] & $97.8 \%$ & $97.8 \%$ & $97.8 \%$ & $97.8 \%$ & 10801 \\
ResNet-152 [44] & $96.4 \%$ & $96.4 \%$ & $96.4 \%$ & $96.4 \%$ & 523 \\
Random Forest [44] & $95.7 \%$ & $95.7 \%$ & $95.7 \%$ & $95.7 \%$ & 573 \\
SVM [44] & $92.2 \%$ & $92.2 \%$ & $92.2 \%$ & $92.2 \% \%$ & 512 \\
AdaBoost [44] & $91.1 \%$ & $91.4 \%$ & $91.1 \%$ & $91.1 \% \%$ & 502 \\
$k$-NN [44] & $92.2 \%$ & $92.2 \%$ & $92.2 \%$ & $92.2 \%$ & 504 \\
Naïve Bayes [44] & & & & & \\
\hline
\end{tabular}

state-of-the-art approaches, including the xDNN approach, DenseNet-201, and ResNet-152. Furthermore, the training time consumed by the xDNN approach was just 210 seconds, or 400 times faster than the Inception-Resnet-v2. The xDNN basis approach consumed just 11.82 seconds to process the task as it is not equipped with any segmentation mechanism, however, the explainablity of the model is affected as it uses the whole image as prototype. The decision structure of the proposed Super-xDNN algorithm is illustrated by Fig. 5. The decisions made by the Super-xDNN method provides highly interpretable insights that may be helpful for medical doctors to audit the results and gain trustworthiness on the network.

Furthermore, the Super-xDNN also allows the creation of a heat-map where the superpixels with highest values of similarities with the prototypes identified for the positive class (Covid-19) are highlighted as illustrated by Fig. 6. The heat-map improves the explainability and understanding of the decisions made by the Super-xDNN network.

Balanced one-way ANalysis Of VAriance (ANOVA) [45] was used to compare the results provided by the classification methods. The null hypothesis is that the mean results provided by the methods are the same. A cutoff value $p$ less than 0.05 suggests that the accuracy of at least one of the algorithms is significantly different from the others. A $p=4.38 e-22$ was obtained and, therefore, the mean accuracy of the algorithms are not all the same; the null hypothesis was rejected.

The Tukey Honestly Significant Difference (HSD) test [45] was performed to compare pairs of classifiers. Table III shows the results of the Tuckey HSD test for a 95\% confidence interval for the true difference of the means.

If the $p-a d j<0.05$ than the null hypothesis is rejected and the difference between the methods are statistically significant. As shown in Table III the proposed Super-xDNN has results statistically different from all the approaches considered in this paper, including the $\mathrm{xDNN}$ basis approach and well known deep learning approaches as DenseNet-201, and ResNet-152.

The Super-xDNN has demonstrated during the experiments that it can be an alternative to "black-box" approaches as it combines highly accurate results with transparent decisions analysis.

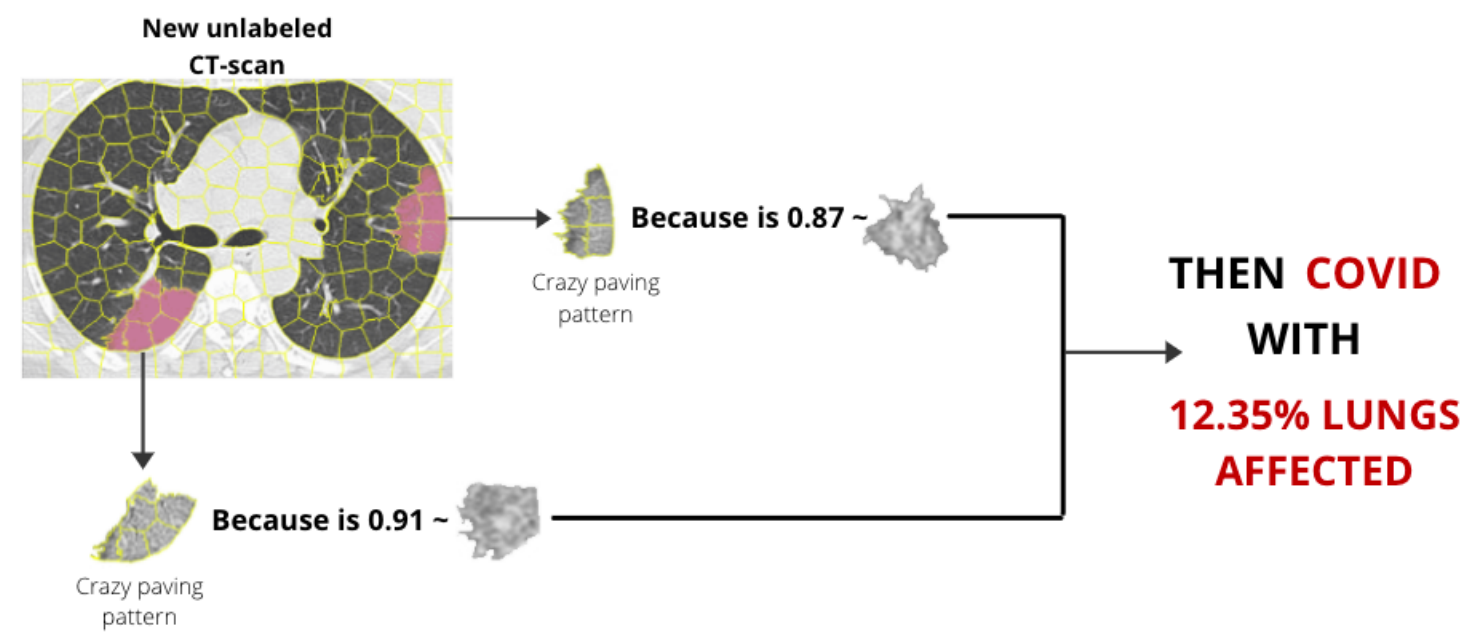

Fig. 5. Decison explanation provided by the Super-xDNN classifier for the COVID-19 identification through superpixels. Differently from typical deep neural networks, the Super-xDNN provides a transparent structure that allows humans to inspect the decisions made by the network. In this case, the CT-scan has been classified as Covid-19 because it presents two crazy paving patterns that are typical from Covid-19. Moreover, the superpixels segmentation also allowed to estimate the area of the lung affected by the disease. 
TABLE III

Tukey Honestly SignificAnt Difference Test

\begin{tabular}{cl|ccccc}
\hline Method 1 & Method 2 & meandiff & p-adj & lower & upper & Statistically different \\
\hline xDNN & Super-xDNN & 1.52 & 0.0032 & -3.3185 & -1.008 & $\underline{\text { True }}$ \\
DenseNet-201 & Super-xDNN & 3.3783 & 0.0015 & -5.7587 & -0.998 & $\underline{\text { True }}$ \\
ResNet-152 & Super-xDNN & 5.6583 & 0.001 & -8.0387 & -3.278 & $\underline{\text { True }}$ \\
Random Forest & Super-xDNN & 2.385 & 0.0493 & -4.7654 & -0.0046 & $\underline{\text { True }}$ \\
SVM & Super-xDNN & 3.7567 & 0.001 & -6.137 & -1.3763 & $\underline{\text { True }}$ \\
Adaboost & Super-xDNN & 15.5983 & 0.001 & -17.9787 & -13.218 & $\underline{\text { True }}$ \\
$k$-NN & Super-xDNN & 15.4933 & 0.001 & -17.8737 & -13.113 & $\underline{\text { True }}$ \\
Naïve Bayes & Super-xDNN & 17.8783 & 0.001 & -20.2587 & -15.498 & $\underline{\text { True }}$ \\
\hline
\end{tabular}

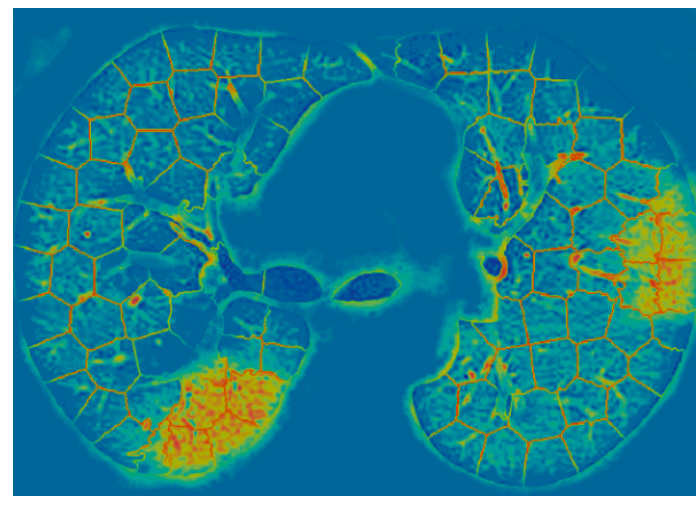

Fig. 6. The heat-map highlights the superpixels with the highest values of similarities with the prototypes that denotes the Covid-19 class.

\section{CONCLUSION}

In this paper, we present a new explainable-by-design approach for Covid-19 classification via CT-scans. During this study, we consider a large dataset for Covid-19 identification via CT-scans that we recently published and attracted the attention to many researchers. The considered dataset is composed of $2482 \mathrm{CT}$ scans slices, out of which 1252 correspond to 60 patients confirmed with Covid-19 and $1230 \mathrm{CT}$ scans slices corresponding to 60 patients with other pulmonary diseases. The proposed approach has an integrated segmentation mechanism composed by a SLIC superpixel technique which allowed a better performance and explanations. The Super-xDNN classifier demonstrates the statically better results in terms of performance than other state-of-the-art approaches, presenting an $F 1$ score of $98.9 \%$ for the proposed task. Moreover, it also provides explanations that may benefit medical doctors to audit the decisions made by the network. It is of great importance for medical specialists to understand and diagnose COVID19 at early stages via computed tomography. In summary, the proposed method demonstrated the following advantages: i)best performance as compared with the top state-of-the-art algorithms; ii) high level of explainability (prototype-based, rule-based, visualisation using actual images); iii) estimation of the area affected by the disease.

\section{REFERENCES}

[1] C. Huang, Y. Wang, X. Li, L. Ren, J. Zhao, Y. Hu, L. Zhang, G. Fan, J. Xu, X. Gu et al., "Clinical features of patients infected with 2019 novel coronavirus in wuhan, china," The Lancet, vol. 395, no. 10223, pp. 497-506, 2020.

[2] N. Zhu, D. Zhang, W. Wang, X. Li, B. Yang, J. Song, X. Zhao, B. Huang, W. Shi, R. Lu et al., "A novel coronavirus from patients with pneumonia in china, 2019," New England Journal of Medicine, 2020.

[3] F. Zhou, T. Yu, R. Du, G. Fan, Y. Liu, Z. Liu, J. Xiang, Y. Wang, B. Song, X. Gu et al., "Clinical course and risk factors for mortality of adult inpatients with covid-19 in wuhan, china: a retrospective cohort study," The Lancet, 2020.

[4] C. Sohrabi, Z. Alsafi, N. O'Neill, M. Khan, A. Kerwan, A. AlJabir, C. Iosifidis, and R. Agha, "World health organization declares global emergency: A review of the 2019 novel coronavirus (covid-19)," International Journal of Surgery, 2020.

[5] A. Lovato and C. De Filippis, "Clinical presentation of covid-19: a systematic review focusing on upper airway symptoms," Ear, Nose \& Throat Journal, vol. 99, no. 9, pp. 569-576, 2020.

[6] M. Ballow and C. L. Haga, "Why do some people develop serious covid-19 disease after infection, while others only exhibit mild symptoms?" The Journal of Allergy and Clinical Immunology: In Practice, vol. 9, no. 4, pp. 1442-1448, 2021.

[7] D. DeCaprio, J. Gartner, T. Burgess, K. Garcia, S. Kothari, S. Sayed, and C. J. McCall, "Building a covid-19 vulnerability index," arXiv preprint arXiv:2003.07347, 2020.

[8] T. Ai, Z. Yang, H. Hou, C. Zhan, C. Chen, W. Lv, Q. Tao, Z. Sun, and L. Xia, "Correlation of chest ct and rt-per testing in coronavirus disease 2019 (covid-19) in china: a report of 1014 cases," Radiology, p. 200642, 2020.

[9] M.-Y. Ng, E. Y. Lee, J. Yang, F. Yang, X. Li, H. Wang, M. M.s. Lui, C. S.-Y. Lo, B. Leung, P.-L. Khong et al., "Imaging profile of the covid-19 infection: radiologic findings and literature review," Radiology: Cardiothoracic Imaging, vol. 2, no. 1, p. e200034, 2020.

[10] W. Kong and P. P. Agarwal, "Chest imaging appearance of covid19 infection," Radiology: Cardiothoracic Imaging, vol. 2, no. 1, p. e200028, 2020.

[11] H. Shi, X. Han, N. Jiang, Y. Cao, O. Alwalid, J. Gu, Y. Fan, and C. Zheng, "Radiological findings from 81 patients with covid-19 pneumonia in whan, china: a descriptive study," The Lancet Infectious Diseases, 2020.

[12] S. Bhattacharya, P. K. Reddy Maddikunta, Q.-V. Pham, T. R Gadekallu, S. R. Krishnan S, C. L. Chowdhary, M. Alazab, and M. Jalil Piran, "Deep learning and medical image processing for coronavirus (covid-19) pandemic: A survey," Sustainable Cities and Society, vol. 65, p. 102589, 2021. [Online]. Available: https://www.sciencedirect.com/science/article/pii/S2210670720308076

[13] M. M. Ahsan, K. D. Gupta, M. M. Islam, S. Sen, M. Rahman, M. S. Hossain et al., "Study of different deep learning approach with explainable ai for screening patients with covid-19 symptoms: Using ct scan and chest X-ray image dataset," arXiv preprint arXiv:2007.12525, 2020.

[14] E. D. Tenda, M. Yulianti, M. Asaf, R. Yunus, W. Septiyanti, V. Wulani, C. W. Pitoyo, C. M. Rumende, and S. Setiati, "The importance of chest ct scan in covid-19: A case series," Acta med indones, vol. 52, no. 1, pp. 68-73, 2020. 
[15] P. Afshar, S. Heidarian, N. Enshaei, F. Naderkhani, M. J. Rafiee, A. Oikonomou, F. B. Fard, K. Samimi, K. N. Plataniotis, and A. Mohammadi, "Covid-ct-md, covid-19 computed tomography scan dataset applicable in machine learning and deep learning," Scientific Data, vol. 8 , no. 1 , pp. 1-8, 2021.

[16] M. S. Hossain, G. Muhammad, and N. Guizani, "Explainable ai and mass surveillance system-based healthcare framework to combat covidi9 like pandemics," IEEE Network, vol. 34, no. 4, pp. 126-132, 2020.

[17] F. Firouzi, B. Farahani, M. Daneshmand, K. Grise, J. S. Song, R. Saracco, L. L. Wang, K. Lo, P. Angelov, E. Soares et al., "Harnessing the power of smart and connected health to tackle covid-19: Iot, ai, robotics, and blockchain for a better world," IEEE Internet of Things Journal, 2021.

[18] E. Soares, P. Angelov, S. Biaso, M. H. Froes, and D. K. Abe, "Sarscov-2 ct-scan dataset: A large dataset of real patients ct scans for sars-cov-2 identification," medRxiv, 2020.

[19] M. H. Hesamian, W. Jia, X. He, and P. Kennedy, "Deep learning techniques for medical image segmentation: achievements and challenges," Journal of digital imaging, vol. 32, no. 4, pp. 582-596, 2019.

[20] M. I. Razzak, S. Naz, and A. Zaib, "Deep learning for medical image processing: Overview, challenges and the future," Classification in BioApps, pp. 323-350, 2018.

[21] G. Litjens, T. Kooi, B. E. Bejnordi, A. A. A. Setio, F. Ciompi, M. Ghafoorian, J. A. Van Der Laak, B. Van Ginneken, and C. I. Sánchez, "A survey on deep learning in medical image analysis," Medical image analysis, vol. 42, pp. 60-88, 2017.

[22] J. Ker, L. Wang, J. Rao, and T. Lim, "Deep learning applications in medical image analysis," Ieee Access, vol. 6, pp. 9375-9389, 2017.

[23] D. S. W. Ting, L. R. Pasquale, L. Peng, J. P. Campbell, A. Y. Lee, R. Raman, G. S. W. Tan, L. Schmetterer, P. A. Keane, and T. Y. Wong, "Artificial intelligence and deep learning in ophthalmology," British Journal of Ophthalmology, vol. 103, no. 2, pp. 167-175, 2019.

[24] A. A.-A. Valliani, D. Ranti, and E. K. Oermann, "Deep learning and neurology: a systematic review," Neurology and therapy, vol. 8 , no. 2 , pp. 351-365, 2019.

[25] K. Munir, H. Elahi, A. Ayub, F. Frezza, and A. Rizzi, "Cancer diagnosis using deep learning: a bibliographic review," Cancers, vol. 11, no. 9 , p. 1235, 2019.

[26] E. Soares, P. Angelov, and X. Gu, "Autonomous learning multiplemodel zero-order classifier for heart sound classification," Applied Soft Computing, vol. 94, p. 106449, 2020.

[27] P. P. Angelov, E. A. Soares, R. Jiang, N. I. Arnold, and P. M. Atkinson, "Explainable artificial intelligence: an analytical review," Wiley Interdisciplinary Reviews: Data Mining and Knowledge Discovery, vol. n/a, no. n/a, p. e1424.

[28] E. Soares, P. P. Angelov, B. Costa, M. P. G. Castro, S. Nageshrao, and D. Filev, "Explaining deep learning models through rule-based approximation and visualization," IEEE Transactions on Fuzzy Systems, vol. 29, no. 8, pp. 2399-2407, 2021.

[29] P. Angelov and E. Soares, "Towards explainable deep neural networks (xdnn)," Neural Networks, vol. 130, pp. 185-194, 2020.

[30] R. Achanta, A. Shaji, K. Smith, A. Lucchi, P. Fua, and S. Süsstrunk, "Slic superpixels," Tech. Rep., 2010.

[31] _ "Slic superpixels compared to state-of-the-art superpixel methods," IEEE transactions on pattern analysis and machine intelligence, vol. 34, no. 11, pp. 2274-2282, 2012.

[32] K. He, G. Gkioxari, P. Dollár, and R. Girshick, "Mask r-cnn," in Proceedings of the IEEE international conference on computer vision, 2017, pp. 2961-2969.

[33] J. Redmon, S. Divvala, R. Girshick, and A. Farhadi, "You only look once: Unified, real-time object detection," in Proceedings of the IEEE conference on computer vision and pattern recognition, 2016, pp. 779788.

[34] A. Krizhevsky, I. Sutskever, and G. E. Hinton, "Imagenet classification with deep convolutional neural networks," in Advances in neural information processing systems, 2012, pp. 1097-1105.

[35] K. Simonyan and A. Zisserman, "Very deep convolutional networks for large-scale image recognition," arXiv preprint arXiv:1409.1556, 2014.

[36] C. Szegedy, W. Liu, Y. Jia, P. Sermanet, S. Reed, D. Anguelov, D. Erhan, V. Vanhoucke, and A. Rabinovich, "Going deeper with convolutions," in Proceedings of the IEEE conference on computer vision and pattern recognition, 2015, pp. 1-9.

[37] K. He, X. Zhang, S. Ren, and J. Sun, "Deep residual learning for image recognition," in Proceedings of the IEEE conference on computer vision and pattern recognition, 2016, pp. 770-778.
[38] C. Szegedy, S. Ioffe, V. Vanhoucke, and A. A. Alemi, "Inception-v4, inception-resnet and the impact of residual connections on learning," in Thirty-first AAAI conference on artificial intelligence, 2017.

[39] I. O. Sigirci, H. Ozgur, and G. Bilgin, "Feature extraction with bidirectional encoder representations from transformers in hyperspectral images," in 2020 28th Signal Processing and Communications Applications Conference (SIU). IEEE, 2020, pp. 1-4.

[40] B. Solmaz, S. M. Assari, and M. Shah, "Classifying web videos using a global video descriptor," Machine vision and applications, vol. 24 no. 7, pp. 1473-1485, 2013.

[41] K. Mizuno, Y. Terachi, K. Takagi, S. Izumi, H. Kawaguchi, and M. Yoshimoto, "Architectural study of hog feature extraction processor for real-time object detection," in 2012 IEEE Workshop on Signal Processing Systems. IEEE, 2012, pp. 197-202.

[42] P. P. Angelov and X. Gu, Empirical approach to machine learning. Springer, 2019.

[43] P. Angelov, Autonomous learning systems: from data streams to knowledge in real-time. John Wiley \& Sons, 2012.

[44] E. Tetila, K. Bressem, G. Astolfi, D. A. Sant'Ana, M. C. Pache, and H. Pistori, "System for quantitative diagnosis of covid-19-associated pneumonia based on superpixels with deep learning and chest ct," 2020.

[45] M. L. McHugh, "Multiple comparison analysis testing in anova," Biochemia medica: Biochemia medica, vol. 21, no. 3, pp. 203-209, 2011.

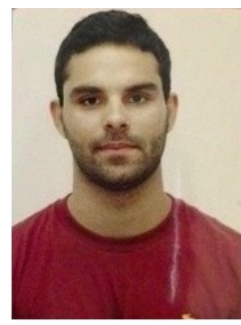

Eduardo Soares Eduardo Soares received his Master's degree from the Federal University of Lavras (Brazil) in 2018. He is currently pursuing his Ph.D. at Lancaster University (UK), where he is funded by Ford Motor Company to work on the project "Transparent Deep Learning Classifier of Driving Scenarios able to Identify and Learn from Unseen Situations" under the supervision of Prof. Plamen Angelov. In 2018, Eduardo received the best student paper award at the IEEE World Conference on Computational Intelligence for the paper "Incremental gaussian granular fuzzy modeling applied to hurricane track forecasting". His research interests include explainable AI, deep neural networks, and deep rule-based classifiers.

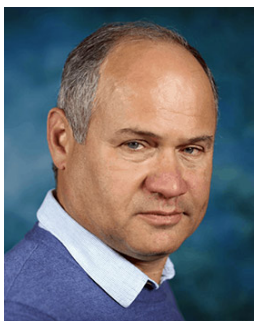

Plamen Angelov Plamen P. Angelov (PhD '93, DSc '15; FIEEE'16) is a Professor/Chair in Intelligent Systems and founding Director of LIRA (Lancaster Intelligent, Robotic and Autonomous systems) Research Centre (www.lancaster.ac.uk/lira ). $\mathrm{He}$ has over 350 publications in leading journals, peer-reviewed conference proceedings, 3 granted US patents, 3 research monographs (by Wiley, 2012 and Springer, 2002 and 2018) cited 116000 times (h-index 55). He has an active research portfolio in the area of explainable AI, including deep neural networks, deep rule-based classifiers as well as anthropomorphic and empirical machine learning and pioneering results in evolving and autonomously learning systems. He holds numerous awards and is the founding co-Editor-in-Chief of Nature-Springer's journal on Evolving Systems and Associate Editor of several leading international journals, including IEEE Transactions on Cybernetics, IEEE Transactions on Fuzzy Systems, etc. He gave over two dozen key note/plenary talks and was General co-Chair of a number of high profile IEEE conferences.

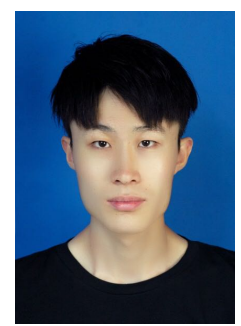

Ziyang Zhang Ziyang Zhang received the B.Sc degree in Information Management and Information System from Beijing Jiaotong University (China), the M.Sc. degree in Data Science from Lancaster University (UK). He is currently working toward the Ph.D. degree in Computer Science a Lancaster University. He is working on an ESAfunded project entitled "Towards explainable AI for Earth Observation (AI4EO): a new frontier to gain trust into the AI" under the supervision of Prof. Plamen Angelov. His research interests include deep learning, remote sensing and explainable AI. 